\title{
Design Elements of Pervasive Games for Elderly Players: A Social Interaction Study Case
}

\section{$\operatorname{AUTHOR}(\mathrm{S})$ :}

Santos, Luciano H.O.; Okamoto, Kazuya; Hiragi, Shusuke; Yamamoto, Goshiro; Sugiyama, Osamu; Aoyama, Tomoki; Kuroda, Tomohiro

\section{CITATION:}

Santos, Luciano H.O. ... [et al]. Design Elements of Pervasive Games for Elderly Players: A Social Interaction Study Case. Lecture Notes in Computer Science 2019, 11593: 204-215

\section{ISSUE DATE:}

2019

URL:

http://hdl.handle.net/2433/243344

\section{RIGHT:}

This is a post-peer-review, pre-copyedit version of an article published in Lecture Notes in Computer Science. The final authenticated version is available online at: http://dx.doi.org/10.1007/978-3-030-22015-0_16; The full-text file will be made open to the public on 8 June 2020 in accordance with publisher's 'Terms and Conditions for Self-Archiving'.; This is not the published version. Please cite only the published version.; この論文は出版社版でありません。引用の際には 出版社版をご確認ご利用ください。 


\title{
Design elements of pervasive games for elderly players: a social interaction study case
}

\author{
Luciano H O Santos ${ }^{1}$, Kazuya Okamoto ${ }^{1,2}$, Shusuke Hiragi ${ }^{1,2}$, Goshiro \\ Yamamoto $^{2}$, Osamu Sugiyama ${ }^{3}$, Tomoki Aoyama ${ }^{4}$, and Tomohiro Kuroda ${ }^{1,2}$ \\ 1 Graduate School of Informatics, Kyoto University, Kyoto, Japan, \\ Ihsantos@kuhp.kyoto-u.ac.jp \\ 2 Division of Medical Information Technology and Administration Planning, Kyoto \\ University Hospital, Kyoto, Japan \\ 3 Preemptive Medicine \& Lifestyle-Related Disease Research Center, Kyoto \\ University Hospital, Kyoto, Japan \\ ${ }^{4}$ Graduate School of Medicine, Kyoto University, Kyoto, Japan
}

\begin{abstract}
We present the design process and evaluation of a pervasive, location-based mobile game created to act as an experiment system and allow evaluation of how different design elements can influence player behaviour, using social interaction as a study case. A feasibility study with a group of community dwelling elderly volunteers from the city of Kyoto, Japan, was performed to evaluate the system. Results showed that the choice of theme and overall design of game was adequate, and that elderly people could understand the game rules and their goals while playing. Points of improvement included reducing the complexity of game controls and changing social interaction mechanics to account for situations when there are only a few players active or players are too far apart.
\end{abstract}

Keywords: elderly, pervasive games, serious games, physical activity, social interaction

\section{Introduction}

Populations are ageing worldwide [1] and societies face an increasing number of challenges to promote quality of life for elderly citizens. Aiming at preserving their health, many studies have employed electronic games as an approach help elderly people lead more active lifestyles, with varied levels of success [2].

In that context, a new family of games that has yet to be fully explored are the so-called pervasive games [3], a term that describes any game that mixes elements from the real world in their mechanics, blurring the edges of the "magic circle" - i.e., the perceived boundaries where playing takes place [4]. For that reason, such games have also been called ubiquitous [5], context aware [6], mixed reality [7] or even trans-reality [8]. The main goal is to create higher levels of immersion and, thus, more fun experiences. Many commercial pervasive games 
became very popular - e.g., Pokémon $\mathrm{GO}^{1}$, Ingress ${ }^{2}$ and Dead Rising $3^{3}-$, illustrating their potential to engage large groups of players.

Not only pervasive games are particularly immersive and engaging for players of all ages, they can be also especially beneficial for elderly players, since two of the most commonly explored design elements in these games are physical location and social relations among users. By inviting players to visit places in the real world, a pervasive game can promote regular physical activity; and by asking these players to interact with other people, it can help prevent social isolation. Both effects are strongly correlated with higher qualify of life among the elderly and lower incidence of age-related diseases [9-13].

One issue commonly found in previous works - not specific to pervasive games, but affecting any game in general targeting the elderly - is that, even though these studies part from the assumption that electronic games can engage people because they are fun, there's still an excessive or even exclusive focus on health benefits, with aspects such as user's motivations to play and sought experiences left to secondary roles. Nonetheless, any study using games should take these aspects into consideration - a deep and meaningful connection with activities of play and fun is an inherent aspect of human nature [14], and elderly people are no exception. By understanding which design principles can generate the most fun and engaging experiences, it is possible to further increase the results of game-based approaches to health promotion.

In this paper, we describe a pervasive game that was designed specifically to test different design elements, and how they affect player behaviour. To evaluate our system, we chose social interaction as study case and performed a feasibility study with community dwelling elderly volunteers from Kyoto city.

The remaining of this text is organized as follows. Section 2 makes a brief literature review of relevant related works. Section 3 describes the design process and the resulting system. Section 4 presents the evaluation method and the results. Finally, Section 5 makes final considerations and lists possible future steps.

\section{Related works}

Pervasive games have emerged as research field in the last decade, with several games made [15] and there are a few works focusing particularly on design [16, 17], while games for elderly became a research topic on its own in the context of serious games for health $[18,19]$.

Some works investigated challenges when designing for older audiences [2022], identifying common physical and cognitive limitations that should be considered. De Schutter et al. point out that older players of electronic games do not form an homogeneous group, but instead, like any other demographic, show

\footnotetext{
${ }^{1}$ Niantic Labs, 2016 - https://www.pokemongo.com/

${ }^{2}$ Niantic Labs, 2013 - https://www.ingress.com/

${ }^{3}$ Microsoft Studios and Capcom, 2013 - https://www.microsoft.com/en-us/p/deadrising-3/
} 
highly varied behaviours and preferences deeply influenced by culture, background and both intrinsic and extrinsic motivations [23]. They propose 5 basic profiles of older players [24] based on the Uses and Gratifications Theory [25] and the Self-Determination Theory [26]. Recent research has started to analyze elements of fun and engagement more deeply [27], following the principle that games should first be fun, and all additional benefits will come later as a natural consequence of playing [28].

A few studies tried to identify preferences of elderly people regarding content and/or genre of the games, primarily using surveys [29-31]. Recent works evaluated elderly players' experiences with different kinds of games, focusing on specific styles of game, such as casual games [32]. A commonly explored genre is the one of exergames, i.e., games in which the player must perform specific kinds of coordinated movements to control the input, sometimes in association with other cognitive tasks [33]. Many studies used varied interfaces for such games, evaluating the user's acceptance of the technology and/or motivation to play $[34,35]$. There are also some examples of pervasive games targeting older adults, usually focusing on specific goals, such as cognitive training [36] and promotion of physical activity using social incentives [37].

\section{Methods}

While previous works using games to help the elderly focused on basically comparing game and non-game strategies $[15,16]$, this research compares variations on the design of the same game. In order to do that, the first problem that must be addressed is how to appropriately test design.

Hebert Simon defines any design problem as a "wicked" or "ill-defined" problem [38], i.e., a problem that cannot be fully understood from start, but must be continuously redefined and worked out via in an iterative process. A good strategy to perform this process is by using an experiment system, a system that rather than immediately solving a problem, focuses on understanding it to evaluate how specific elements can change the outcome. In the next sections, we describe the design process we followed to create such a system.

\subsection{Design principles}

If the game's purpose is to act as an experiment system, it should not only be pervasive, but also allow for easy contextual adaptations in its pervasive mechanics. One way of framing this problem is by using Schell's elemental tetrad (Fig. 1), that describes a game in terms of four domains that affect each other and combine to create specific experiences to the player.

A pervasive game is one that integrates elements from the real world into one or more of these domains [17], thus, a pervasive game that is also an appropriate experiment system should allow for changes on these integrations that can be isolated and controlled for testing. 


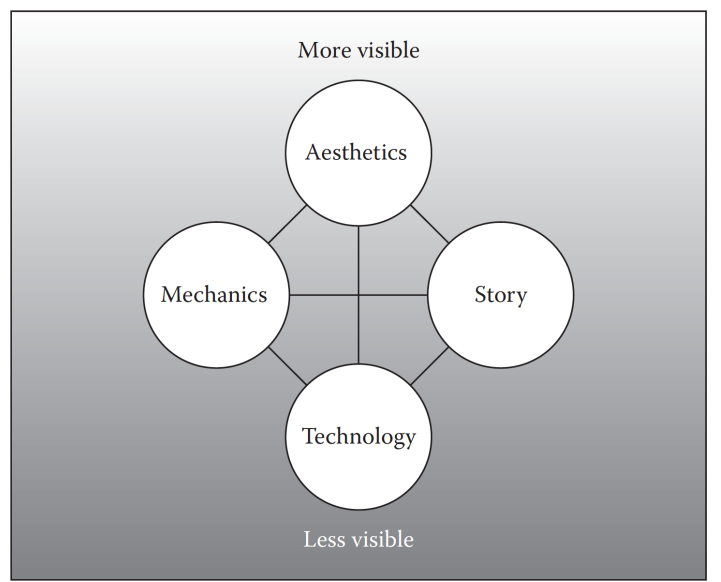

Fig. 1: Schell's elemental tetrad [39]: aesthetics - all parts of the game accessible to the player, such as visuals, sounds and controls; mechanics - all game rules, valid states and constraints; story - the elements of narrative; technology set of physical components that allow the game to take place.

Taking on this idea as a guide, we used an iterative design process that analyzed existing (pervasive) games and took the input of specialists from varied fields and from final users, to lay out general design principles:

- the main goal is collection of items - this is a play element present in almost every culture and appreciated by players of varied ages, backgrounds and motivations; since the main point is the abstraction of collecting items, the nature of the items can be easily changed; this allows for adaptations on:

- aesthetics - different representations and visual styles;

- story - different meanings of the items in the game narrative;

- technology - different media, such as virtual or physical items;

- items are collected by walking to specific locations - this serves the research goal of promoting physical activity, but also allows for flexibility, with variations on:

- mechanics - varied constraints of navigation and access to places;

- story - different meanings and roles of places in the game narrative;

- technology - different types and arrangements of places, as well as levels of scale (e.g., within a room, a building, a city);

- players should be able to collaborate for mutual benefit and the game should use positive reinforcement to promote pro-social behaviour - even though social interaction in the form of player vs player competition is enjoyed by some people, collaboration can be enjoyed by any person; this allows for variations on:

- mechanics, technology - direct vs virtual, static vs casual, known people vs strangers, groups vs individuals, etc...; 
- story - different roles of people in the game narrative.

Based on these design principles, we developed Shinpo - in Japanese, 神 歩, meaning “sacred steps" -, a pervasive location-based mobile game targeting Japanese elderly people. The next section describes the game.

\subsection{Game design}

In Shinpo, players must to collect virtual cards (Fig. 2) by walking and visiting real locations - temples and shrines - around Kyoto city. Each card has an animal (from Japanese fauna or folklore) and a level, indicated by its color (1 violet, 2 - green, 3 - blue, 4 - gold).
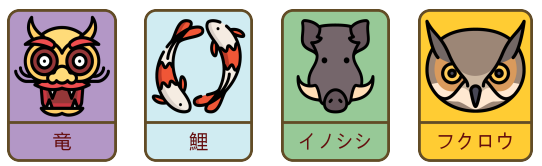

Fig. 2: Examples of game cards.

Using the map (Fig. 3(a)), players can locate nearby hotspots and receive some card by visiting them. Cards are earned the first time within a day that the player enters a shrine and periodically depending on how much they walked and how many hotspots they visited on the previous days.

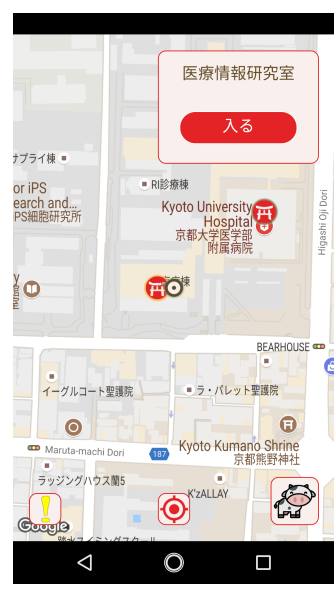

(a) world map

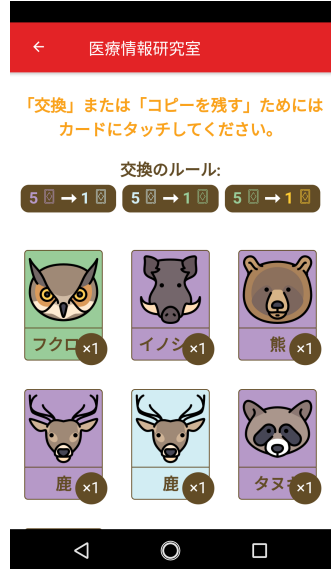

(b) hotspot menu

Fig. 3: Some screenshots from Shinpo. 
Inside the hotspot, players can see their current cards (Fig. 3(b)) and trade a certain number of cards of a level for one card of the next level. Because there are 4 levels, it takes a long time to achieve the game's goal of having all the possible gold cards. By design, players with higher levels of physical activity can win the game faster.

Shinpo uses a specific theme to appeal to Japanese (elderly) people - shrines and card game references -, but, as explained in Section 3.1, its design principles allow for variations of these choices, while keeping the core elements.

For instance, other abstractions could be used (e.g., zodiac signs instead of animals or coins instead of cards); different rules and interaction strategies (e.g., cooperation, competition, challenges, hierarchies); different visual styles (e.g., traditional, cute, cartoonish); and different technologies (e.g., physical objects, $I o T$ devices). These adaptations, when limited within a closed context, could also be presented to different groups of players to evaluate their specific experience. As an example, the color schemes or the illustration style could be changed and evaluated for their appeal to different audiences.

To test the adaptability of the game, we proposed a variation using including social interaction. The proposed variation includes these additional rules:

- players have customized profiles that identify them to other players within the game and also see and "like" other players'actions;

- players can, once per day, leave a card at a hotspot; other players will be notified about it and will receive a copy when they enter that hotspot; the owner is rewarded with additional random cards of same level based on the number of total copies distributed this way;

- everyday, players receive a challenge of visiting a certain number of shrines in collaboration with other players, if they join the challenge and reach their goal, all members of the group can get more cards;

- nearby players can meet in person, and receive cards by doing so.

\section{Evaluation}

To evaluate the game, we performed a 3 -week feasibility study (Fig. 4) with volunteer community dwelling senior citizens. Participants were recruited from a group of people who attend a program run by Kyoto University Hospital, offering weekly sessions of exercise-based cognitive training at a local community center.

At the beginning of the study, participants answered a questionnaires about their previous experience with technology (if they have and use smartphones and/or personal computers, how often they use them and what kind of tasks they use them for) and with games in general and electronic games specifically (what kinds of games they play, how often, using which devices and with whom). No previous experience was required to join the experiment, volunteers received a pre-configured smartphone and were given an explanation about its basic operation.

During the first week, subjects were asked to simply carry the smartphone around with them, to register their baseline level of physical activity, measured 


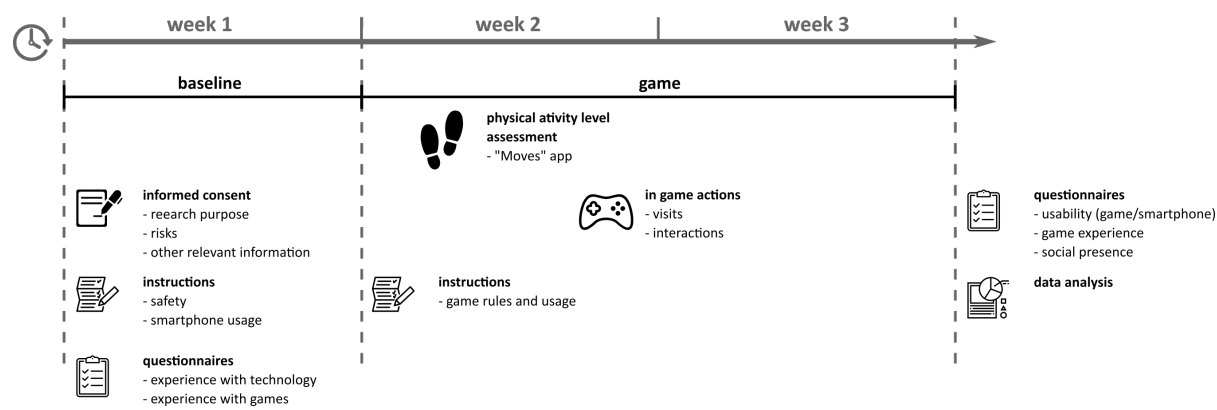

Fig. 4: Feasibility study protocol.

by the number of steps as calculated using the smartphone sensor data. On the following 2 weeks, they played the game, while their step count continued to be monitored.

At the end of the study, volunteers were asked to answer questionnaires to assess the usability of the game, and to report their experience during the game, including their sense of social presence. The questions were based and/or adapted from the Game Experience Questionnaire (GEQ) [40] and translated to Japanese by native speakers. All questions had a 5-level Likert scale of one of two types: agreement level $(0=$ strongly disagree, $4=$ strongly agree $)$ and frequency ( $0=$ never, $4=$ always $)$.

\subsection{Results}

In Table 1, basic demographic data about the participants is presented. The average scores of the usability, game experience and social presence evaluation are presented in Table 2 .

Results indicated that the proposed design was successful in appealing to Japanese elderly people. Users could learn the basic operation of the smartphone without problems, even if most of them had never used one before. Players were able to understand the game rules and goals and overall liked its visual style. There were mixed results about learning the game controls.

Players also enjoyed the challenge level of the game and also reported engagement and satisfaction/motivation to play and enjoyment/fulfillment. There was a general sense that the game stimulates players to explore their surroundings and discover new things, which was also corroborated by some open questions comments. There was strong approval of the game theme (Japanese shrines).

The Social Presence Questionnaire section of GEQ had overall low scores for all items. We hypothesized that this was due to the small number of participants, i.e., the proposed mechanics require a large number of simultaneous players to be effective, thus a revision of such mechanics is necessary for the next steps of the research. 
Table 1: Participants' demographic data and previous experience technology and games.

\begin{tabular}{|l|r|}
\hline \multicolumn{2}{|c|}{ Demographics } \\
\hline Average age $(\sigma)$ & $74.6(3.3)$ \\
Number of participants & 12 \\
Female & $9(75 \%)$ \\
Dropouts & $3(\mathrm{~F}=2)$ \\
\hline \multicolumn{2}{|c|}{ Experience with PCs } \\
\hline Frequency of usage & $1(8 \%)$ \\
$\quad$ Everyday & $5(42 \%)$ \\
2+ times/week & $1(8 \%)$ \\
1- times/week & $5(42 \%)$ \\
Never & $1(8 \%)$ \\
Skills & $3(25 \%)$ \\
\hline Internet, email & $5(42 \%)$ \\
Social networks & $6(50 \%)$ \\
\hline Experience with smartphones \\
\hline Never used & $6(50 \%)$ \\
Make calls & $3(20 \%)$ \\
Internet, e-mail & $3(20 \%)$ \\
\hline Social networks
\end{tabular}

\begin{tabular}{|l|r|}
\hline \multicolumn{2}{|c|}{ Non-electronic games } \\
\hline Doesn't play & $9(75 \%)$ \\
\hline Everyday & $0(0 \%)$ \\
2+ times/week & $2(17 \%)$ \\
1- times/week & $1(8 \%)$ \\
\hline
\end{tabular}

\section{Electronic games}

Frequency of play

\begin{tabular}{l|l} 
Doesn't play & $9(75 \%)$
\end{tabular}

Everyday $\quad 1(8 \%)$

$2+$ times/week $\quad 1(8 \%)$

1- times/week

$1(8 \%)$

Partners

(\% of those who play)

Alone

$3(100 \%)$

Family (adults) $1(33 \%)$

Family (children) $\quad 0(0 \%)$

Friends

$0(0 \%)$

Strangers

$0(0 \%)$

Table 2: Usability, game experience, and social presence average scores.

\begin{tabular}{|l|r|}
\hline \multicolumn{1}{|c|}{ Category } & Score \\
\hline Usability & 1.50 \\
Controls & 1.90 \\
Learn curve & 2.25 \\
Feedback & 2.06 \\
Game rules & \\
\hline Game experience & 2.45 \\
Theme and visual style & 2.17 \\
Originality & 2.02 \\
Feeling of Immersion & 2.40 \\
Feeling of Enjoyment & 2.21 \\
Feeling of Engagement & 1.78 \\
Feeling of Freedom/Ability to explore & 2.11 \\
Feeling of (positive) Challenge & 0.48 \\
\hline Social presence &
\end{tabular}


Even though the sample was too small for any further analysis and the feasibility stage didn't focus on evaluating the main outcome yet, a slight positive effect in comparison to the baseline was observed (Table 3).

Table 3: Number of steps of the whole group during the study.

\begin{tabular}{|r|c|r|}
\hline Week & Mean $(\sigma)$ & Effect (relative to $\sigma$ ) \\
\hline 1 & $22567.2(16347.8)$ & \\
\hline 2 & $24272.9(16995.3)$ & $+1705.7(7.6 \%)$ \\
\hline 3 & $24393.3(14203.8)$ & $+1826.1(8.1 \%)$ \\
\hline
\end{tabular}

\section{Conclusion}

In this paper, we presented the design process and implementation of an experiment system that allows for testing of variations in pervasive game design targeting elderly people and evaluate their effect on player's behaviour.

The results indicate that the choice of theme and visual style for the proposed game was adequate, and that elderly people could understand the rules and goals of the game. Participants also reported feeling challenged and engaged, but there might be difficulties when it comes to learning complex controls.

The proposed social interaction mechanics weren't very effective. We believe this happened because the proposed mechanics require a large number of simultaneous players to be effective, thus a revision of such mechanics taking these findings into account is necessary for future iterations.

A deeper understanding of how pervasive mechanics affect older audiences can be a powerful asset for researchers and designers aiming at using pervasive games to promote the well-being of elderly people. In our future steps, we will improve our design to address the issues identified in our study, and investigate other variations on design, aiming at further expanding our understanding of how design choices can affect player experience and behaviour.

\section{References}

1. UN: World Population Ageing. Technical report, United Nations, Department of Economic and Social Affairs, Population Division (2017)

2. Bleakley, C.M., Charles, D., Porter-Armstrong, A., McNeill, M.D.J., McDonough, S.M., McCormack, B.: Gaming for Health: A Systematic Review of the Physical and Cognitive Effects of Interactive Computer Games in Older Adults. Journal of Applied Gerontology 34(3) (apr 2013) 0733464812470747-0733464812470747-

3. Kasapakis, V., Gavalas, D.: Pervasive gaming: Status, trends and design principles. Journal of Network and Computer Applications 55 (sep 2015) 213-236

4. Montola, M.: Exploring the edge of the magic circle: Defining pervasive games. Proceedings of DAC 1966 (2005) 16-19 
5. Björk, S., Holopainen, J., Ljungstrand, P., Akesson, K.P., Staffan Björk, Jussi Holopainen, Peter Ljungstrand, K.P.Å.: Designing Ubiquitous Computing Games: A Report from a Workshop Exploring Ubiquitous Computing Entertainment. Personal Ubiquitous Comput. 6(5-6) (2002) 443-458

6. Koskinen, K., Suomela, R.: Rapid prototyping of context-aware games. In: 2nd IET International Conference on Intelligent Environments (IE 06). Volume 2006., IEE (2006) v1-135-v1-135

7. Bonsignore, E.M., Hansen, D.L., Toups, Z.O., Nacke, L.E., Salter, A., Lutters, W.: Mixed reality games. In: Proceedings of the ACM 2012 conference on Computer Supported Cooperative Work Companion - CSCW '12, New York, New York, USA, ACM Press (2012) 7

8. Gutierrez, L., Stroulia, E., Nikolaidis, I.: fAARS: A Platform for Location-Aware Trans-reality Games. In: Proceedings of the 11th International Conference on Entertainment Computing. ICEC'12. Springer-Verlag, Berlin, Heidelberg (2012) $185-192$

9. Colcombe, S., Kramer, A.F.: Fitness Effects on the Cognitive Function of Older Adults. Psychological Science 14(2) (mar 2003) 125-130

10. Larsen, L.H., Schou, L., Lund, H.H., Langberg, H.: The Physical Effect of Exergames in Healthy Elderly-A Systematic Review. Games for Health Journal 2(4) (aug 2013) 205-212

11. Vagetti, G.C., Barbosa Filho, V.C., Moreira, N.B., de Oliveira, V., Mazzardo, O., de Campos, W.: Association between physical activity and quality of life in the elderly: a systematic review, 2000-2012. Revista Brasileira de Psiquiatria 36(1) (jan 2014) 76-88

12. Tomaka, J., Thompson, S., Palacios, R.: The Relation of Social Isolation, Loneliness, and Social Support to Disease Outcomes Among the Elderly. Journal of Aging and Health 18(3) (jun 2006) 359-384

13. Lee, G.R., Ishii-Kuntz, M.: Social Interaction, Loneliness, and Emotional WellBeing among the Elderly. Research on Aging 9(4) (dec 1987) 459-482

14. Huizinga, J.: Homo Ludens: A Study of Play-element in Culture. Routledge (1949)

15. Buzeto, F.N.F., Castillo, A.H.O.R., Castanho, C.D., Jacobi, R.P.: What is going on with ubicomp games. In: XI Brazilian Symposium on Games and Digital Entertainment, Brasília, DF, Brazil, Universidade de Bras\'ilia, SBC (2012) 1-7

16. Kasapakis, V., Gavalas, D., Bubaris, N.: Pervasive Games Research: A Design Aspects-Based State of the Art Report. In: Proceedings of the 17th Panhellenic Conference on Informatics - PCI '13, New York, New York, USA, ACM Press (2013) 152

17. Buzeto, F.N., Silva, T.B.P.e., Castanho, C.D., Jacobi, R.P.: Reconfigurable Games: Games that Change with the Environment. In: 2014 Brazilian Symposium on Computer Games and Digital Entertainment, IEEE (nov 2014) 61-70

18. Smith, S.T., Talaei-Khoei, A., Ray, M., Ray, P.: Electronic Games for Aged Care and Rehabilitation. In: 2009 11th International Conference on e-Health Networking, Applications and Services (Healthcom), IEEE (dec 2009) 42-47

19. Garcia Marin, J., Felix Navarro, K., Lawrence, E.: Serious Games to Improve the Physical Health of the Elderly: A Categorization Scheme. International Conference on Advances in Human-oriented and Personalized Mechanisms, Technologies, and Services, CENTERIC 2011 (c) (2011) 64-71

20. Ijsselsteijn, W., Nap, H.H., de Kort, Y., Poels, K.: Digital game design for elderly users. In: Proceedings of the 2007 conference on Future Play - Future Play '07. Future Play '07, New York, New York, USA, ACM Press (2007) 17 
21. Ogomori, K., Nagamachi, M., Ishihara, K., Ishihara, S., Kohchi, M.: Requirements for a Cognitive Training Game for Elderly or Disabled People. In: 2011 International Conference on Biometrics and Kansei Engineering, IEEE (sep 2011) $150-154$

22. Barnard, Y., Bradley, M.D., Hodgson, F., Lloyd, A.D.: Learning to use new technologies by older adults: Perceived difficulties, experimentation behaviour and usability. Computers in Human Behavior 29(4) (jul 2013) 1715-1724

23. De Schutter, B., Brown, J.A., Vanden Abeele, V.: The domestication of digital games in the lives of older adults. New Media \& Society 17(7) (aug 2015) 11701186

24. De Schutter, B., Malliet, S.: The older player of digital games: A classification based on perceived need satisfaction. Communications 39(1) (jan 2014) 67-88

25. Katz, E., Blumler, J., Gurevitch, M.: The uses of mass communication:Current Perspectives on Gratifications Research. Sage Publications (1974)

26. Ryan, R., Deci, E.: Self-determination theory and the facilitation of intrinsic motivation. American Psychologist 55(1) (2000) 68-78

27. Gerling, K., De Schutter, B., Brown, J., Allaire, J.: Ageing Playfully: Advancing Research on Games for Older Adults Beyond Accessibility and Health Benefits. In: Proceedings of the 2015 Annual Symposium on Computer-Human Interaction in Play. CHI PLAY '15, New York, NY, USA, ACM (2015) 817-820

28. De Schutter, B., Vanden Abeele, V.: Towards a Gerontoludic Manifesto. Anthropology \& Aging 36(2) (nov 2015) 112-120

29. Carvalho, R.N.S.D., Ishitani, L., Nogueira Sales De Carvalho, R., Ishitani, L.: Motivational Factors for Mobile Serious Games for Elderly Users. In: XI Brazilian Symposium on Games and Digital Entertainment, Brasília, DF, Brazil, Universidade de Brasília, SBC (2012) 19-28

30. Cota, T.T., Ishitani, L., Vieira, N.: Mobile game design for the elderly: A study with focus on the motivation to play. Computers in Human Behavior 51 (oct 2015) 96-105

31. Kaufman, D., Sauve, L., Renaud, L., Sixsmith, A., Mortenson, B.: Older Adults Digital Gameplay: Patterns, Benefits, and Challenges. Simulation \& Gaming 47(4) (aug 2016) 465-489

32. Chesham, A., Wyss, P., Müri, M.R., Mosimann, P.U., Nef, T.: What Older People Like to Play: Genre Preferences and Acceptance of Casual Games. JMIR Serious Games 5(2) (apr 2017) e8

33. Kayama, H., Okamoto, K., Nishiguchi, S., Yamada, M., Kuroda, T., Aoyama, T.: Effect of a Kinect-Based Exercise Game on Improving Executive Cognitive Performance in Community-Dwelling Elderly. Journal of Medical Internet Research 16(2) (feb 2014) e61

34. Brox, E., Konstantinidis, T.S., Evertsen, G.: User-Centered Design of Serious Games for Older Adults Following 3 Years of Experience With Exergames for Seniors: A Study Design. JMIR Serious Games 5(1) (jan 2017) e2

35. Meekes, W., Stanmore, K.E.: Motivational Determinants of Exergame Participation for Older People in Assisted Living Facilities: Mixed-Methods Study. J Med Internet Res 19(7) (jul 2017) e238

36. Gamberini, L., Martino, F., Seraglia, B., Spagnolli, A., Fabregat, M., Ibanez, F., Alcaniz, M., Andres, J.M.: Eldergames project: An innovative mixed reality table-top solution to preserve cognitive functions in elderly people. In: 2009 2nd Conference on Human System Interactions, IEEE (may 2009) 164-169 
37. Brox, E., Fernandez-Luque, L., Evertsen, G., González-Hernández, J.: Exergames For Elderly: Social exergames to persuade seniors to increase physical activity. In: Proceedings of the 5th International ICST Conference on Pervasive Computing Technologies for Healthcare, IEEE (2011) 546-549

38. Simon, H.A.: The Sciences of the Artificial. 1st edn. MIT Press, Cambridge, MA, USA (1969)

39. Schell, J.: The Art of Game Design: A Book of Lenses. Morgan Kaufmann Publishers Inc., San Francisco, CA, USA (2008)

40. Poels, K., de Kort, Y.A.W., Ijsselsteijn, W.A.: D3.3 : Game Experience Questionnaire. Technical report, Technische Universiteit Eindhoven, Eindhoven (2007) 